Methods Men with urethritis, women with PID and current sexual partners of Mgen-infected patients were tested for Mgen (BASHH guidelines). The samples were tested using the Fast-Track Urethritis Basic assay for detection. Positive samples were tested by the SpeeDx ResistancePlus ${ }^{\circledR}$ MG assay to detect the presence of MR-mutations and the SpeeDx MG + ParC (beta) assay determined QR-mutations.

Results Forty-five patients tested positive for Mgen-53\% of cases were men with urethritis; 29\% were women with PID and $18 \%$ were asymptomatic patients. The prevalence of Mgen in men with urethritis was $18 \%$, and in women with PID was also 18\%. The prevalence of MR was 69\% (31/45). The prevalence of QR was 7\% (3/45); all 3 patients also had MR.

Conclusion These are the first UK data for $M R$ and $Q R$ in Mgen from attendees to clinic at a single centre. MR was higher than previously reported in the UK and Europe. Reassuringly, QR is still low-however, this is likely to rise with increasing quinolone use. Patients with dual-class resistance pose a significant challenge as subsequent treatment options are limited. All testing for Mgen should include the detection of resistance-associated mutations so that the most appropriate agent can be used.

Disclosure No significant relationships.

\section{P620 INCLUSIVITY, EXCLUSIVITY, STABILITY AND PROSPECTIVE TESTING OF TWO REAL-TIME PCR ASSAYS FOR MYCOPLASMA GENITALIUM}

\begin{abstract}
${ }^{1} J u s t i n$ Hardick, ${ }^{2}$ Litty Tan, ${ }^{2}$ Cassandra Ingles, ${ }^{2}$ Colin Denver, ${ }^{3}$ Barbara Van Der Pol, ${ }^{4}$ Jorgen Jensen, ${ }^{5}$ Maria Trent, ${ }^{6}$ Charlotte Gaydos. ${ }^{1}$ Johns Hopkins University School of Medicine, Infectious Diseases, Baltimore, USA; ${ }^{2}$ SpeeDx Pty Ltd., Sydney, Australia; ${ }^{3}$ University of Alabama at Birmingham, Medicine/Infectious Diseases, Birmingham, USA Minor Outlying Islands; ${ }^{4}$ Statens Serum Institut, DK, Denmark; ${ }^{5}$ Johns Hopkins University School of Medicine, Ped Gen Pediatrics Adoles Medicine, Baltimore, USA; ${ }^{6}$ Johns Hopkins University, Division of Infectious Diseases, Baltimore, USA
\end{abstract}

\subsection{6/sextrans-2019-sti.688}

Background Mycoplasma genitalium (MG) is an emerging sexually transmitted infection. It has been associated with cervicitis and PID in women and urethritis and persistent NGU in men.

Methods We evaluated two MG qPCRs, 16S rRNA and pdhD. The limit of detection (LOD) for the 16S rRNA and pdhD assays were determined with $11 \mathrm{MG}$ strains. Inclusivity/exclusivity testing was performed with 11 MG strains and 43 nonMG strains. Stability testing was performed with mock vaginal and urine samples stored at $+4^{\circ} \mathrm{C}$ and $25^{\circ} \mathrm{C}$ at $1.5 \mathrm{X}, 4 \mathrm{X}$, $10 \mathrm{X}$ and $20 \mathrm{X}$ LOD at $0,7,14,21,28$ and 35 days. These assays were employed in an ongoing prospective study examining the prevalence of MG in symptomatic and asymptomatic men and women. Positives were sequenced to determine mutation rates in the $23 \mathrm{~S}$ rRNA gene conferring macrolide resistance.

Results The pdhD and 16S assays had LODs of 1324 and $1536 \mathrm{copies} / \mathrm{ml}$, respectively. All inclusivity/exclusivity testing performed as expected. Detection in urine and vaginal matrix at $4^{\circ} \mathrm{C}$ was $100 \%$ for both assays. Detection in urine at $4^{\circ} \mathrm{C}$ was $100 \%$ for both assays while detection in urine at $25^{\circ} \mathrm{C}$ was $100 \%$ at 28 days, but was $90 \%$ at 35 days. For symptomatic men, the prevalence was $19 \%(4 / 21)$ and $14.3 \%(3 / 21)$ for the pdhD and 16S rRNA assays respectively, and was $7.14 \%(1 / 15)$ in symptomatic women for both assays. There were no MG detections in asymptomatic subjects. Of the positives, $100 \%(5 / 5)$ were determined to be $23 \mathrm{~S}$ mutants.

Conclusion Both assays had reasonable LODs and expected results for inclusivity/exclusivity testing. For stability testing, results were as expected up to 35 days, where a loss of positivity was observed for urine samples. We observed a high prevalence of MG in symptomatic men and women, as well as a high percentage of $23 \mathrm{~S}$ mutants conferring macrolide resistance.

Disclosure No significant relationships.

\section{P621 PREVALENCE OF MYCOPLASMA GENITALIUM INFECTION, ANTIMICROBIAL RESISTANCE, AND SYMPTOM RESOLUTION FOLLOWING TREATMENT}

${ }^{1}$ Laura Bachmann*, ${ }^{2}$ Robert Kirkcaldy, ${ }^{3}$ William Geisler, ${ }^{4}$ Harold Wiesenfeld, ${ }^{5}$ Lisa Manhart, ${ }^{6}$ Stephanie Taylor, ${ }^{7}$ Arlene Seña, ${ }^{8}$ Candice Mcneil, ${ }^{9}$ Noelle Myler, ${ }^{2}$ Katherine Bowden, ${ }^{9}$ Rachael Fuchs. ${ }^{1}$ Centers for Disease Control and Prevention, Division of STD Prevention, Atlanta, USA; ${ }^{2}$ Centers for Disease Control and Prevention, Atlanta, USA; ${ }^{3}$ University of Alabama at Birmingham, Birmingham, USA; ${ }^{4}$ University of Pittsburgh/Magee-Womens Hospital, Pittsburgh, USA; ${ }^{5}$ University of Washington, Epidemiology, Seattle, USA; ${ }^{6}$ Louisiana State University, Department of Health Sciences, New Orleans, USA; ${ }^{7}$ University of North Carolina at Chapel Hill, Division of Infectious Diseases, Chapel Hill, USA; ${ }^{8}$ Wake Forest Baptist Medial Center, Internal Medicine-Infectious Disease, Winston-Salem, USA; ${ }^{9}$ FHI360, Durham, USA

\subsection{6/sextrans-2019-sti.689}

Background Mycoplasma genitalium (MG) is an emerging cause of urethritis. Although an FDA-approved MG diagnostic test is now available in the U.S., syndromic management of urethritis remains widespread. Little is known about the geographic distribution of MG resistance in the U.S. and associated clinical outcomes. We evaluated the frequency of $\mathrm{MG}$ among men with urethritis, antimicrobial susceptibility of MG, and post-treatment symptom persistence.

Methods We enrolled men presenting with urethritis symptoms to 6 U.S. STD clinics during June 2017-July 2018. Participants with urethritis confirmed on stained urethral smear were eligible for a follow-up phone call 14-17 days post-enrollment and chart review. Urethral specimens were tested locally for N. gonorrhoeae and C. trachomatis. CDC tested specimens for MG and T. vaginalis. MG resistance mutations were detected by targeted amplification/Sanger sequencing of $23 \mathrm{~S}$ rRNA loci (macrolide resistance mutations [MRM]) and parC and gyrA (quinolone resistance mutations).

Results Among 914 participants with evaluable MG results, MG was detected in 28.7\% (95\% CI 23.8-33.6). Men with MG were more often black $(79.8 \%$ vs $66 \%),<30$ years (72.9\% vs $56.2 \%)$, and reported only female partners $(83.7 \%$ vs $74.2 \%$ ) than men without MG. Among MG+ participants, MRM was detected in $62.2 \%$ (range 53\%-72.3\%), parC mutations in $11.5 \%$ (range 6.6-18.4\%), and gyrA in $0 \%$. Among 763 participants with follow-up, 19.8\% reported symptom persistence, without clinically meaningful difference by MG status. Among MG participants treated with azithromycin, those with MRM more often reported persistent symptoms $(35.1 \%)$ and were more likely to return to clinic within 45 days than those without MRM.

Conclusion MG was common among men with urethritis and MRM prevalence high. Persistent symptoms were frequent among men with and without MG. Many participants with macrolide-resistant MG experienced symptom persistence and returned to clinic for evaluation. 
Disclosure No significant relationships.

\section{P622 THE USE OF SEEGENE'S ALLPLEX'MMMG \& AZIR ASSAY FOR THE DETECTION OF MYCOPLASMA GENITALIUM AND MACROLIDE RESISTANCE IN WALES}

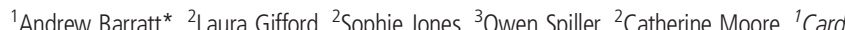
University, Medical Microbiology, Cardiff, UK; ${ }^{2}$ Public Health Wales, Medical Microbiology, Cardiff, UK; ${ }^{3}$ Cardiff University School of Medicine, Medical Microbiology, Cardiff, UK

10.1136/sextrans-2019-sti.690

Background Mycoplasma genitalium (Mgent) is a sexually transmitted bacteria, associated with cervicitis and pelvic inflammatory disease in women and non-gonococcal urethritis in men. These bacteria lack cell walls and many prokaryotic metabolic pathways, mediating inherent resistance to most antimicrobials. Furthermore, Mgent has garnered concern as the prevalence of both fluroquinolone and macrolide resistance has increased significantly in recent years, further restricting possible therapeutic avenues. In January 2019, Public Health Wales deployed the Seegene Allplex ${ }^{\mathrm{TM}} \mathrm{MG} \&$ AziR assay to determine the presence of $\mathrm{MG}$ and its susceptibility to macrolides from genitourinary samples. This kit is novel in its ability to not only detected MG, but also define which specific 23S rRNA gene macrolide-resistance mediating mutations (MRM) are present without requirement for sequencing.

Methods 170 clinical samples (collected Jan-March 2019) were investigated: 83 clinical samples submitted from symptomatic patients (suspected MG infections by BASHH guidelines) combined with 87 samples randomly selected from clinical samples submitted for Cobas gonorrhoea/chlamydia (NG/CT) testing (non-targeted). All samples were from patients attending a genitourinary medicine (GUM) clinic in South Wales. Samples were extracted and prepared using the Hamilton Microlab Nimbus, STARMag universal cartridge extraction kit and Allplex ${ }^{\mathrm{TM}} \mathrm{MG} \&$ AziR assay. Amplification and detection were performed by a Bio-Rad CFX96 equiped with SeeGene interpretative software.

Results Mgent prevalence with suspected NG/CT patients was 5/87 (5.7\%) with 4 (80\%) containing MRM (2x A2058G and 2x A2059G), while prevalence within the Mgent-suspected group was 11/83 (13.3\%) with 5 (45.5\%) containing MRM (4x A2059G and 1x A2058G mutations). Further up-to-date cumulative data to be presented at IUSTI.

Conclusion Mgent prevalence was $5.7 \%$ in the non-targeted cohort, while targeted patients gave $13.3 \%$ prevalence for a South Wales GUM clinic. Macrolide resistance prevalence was $56 \%$ on average. These results justify the implementation of routine Mgent and macrolide resistance testing in South Wales, abiding by European and BASHH guidelines.

Disclosure No significant relationships.

\section{P623 POST-TRANSCRIPTIONAL REGULATION OF GENES BY NON-CODING RNA IN NEISSERIA GONORRHOEAE, AN OBLIGATE HUMAN PATHOGEN}

\footnotetext{
1,2Pooja Tanwer*, 'Daman Saluja. 'University of Delhi, Dr. B.r. Ambedkar Center for Biomedical Research, Delhi, India; ${ }^{2}$ Indian Institute of Technology, Delhi, India
}

10.1136/sextrans-2019-sti.691

Background Small non-coding RNAs (sRNAs) play an important role in bacterial gene expression and regulation. The knowledge of sRNA in Neisseria gonorrhoeae is scarce despite of its clinical significance. We utilized the available RNA-seq data under aerobic and anaerobic condition to identify noncoding RNA. We further identified sRNAs which are differentially expressed under anaerobic condition and their mRNA targets.

Methods The normalized reads (RPKM) under aerobic and anaerobic conditions were compared and a three-fold or greater difference in the expression level of sRNAs was scored as differentially expressed sRNA. sRNA targets were found using online available tools (CopraRNA, targetRNA2). We further predicted the sRNA-mRNA interactions using intaRNA tool.

Results A total of 26 sRNAs were identified. Out of which, ten sRNAs were differentially expressed under anaerobic condition, physiologically important stage during infection. We further identified mRNA targets of these sRNAs based on deep sequencing of $N$. gonorrhoeae transcriptome under aerobic and anaerobic conditions. These results indicated that several sRNAs target genes that are involved in energy metabolism processes, stress response and various other networks.

Conclusion Our results provide new insights into the posttranscriptional regulation of genes by sRNAs in Neisseria gonorrhoeae.

Disclosure No significant relationships.

\section{P624 CULTURE FOR URETHRAL GONORRHEA FROM ASYMPTOMATIC MEN POSITIVE FOR NEISSERIA GONORRHOEAE BY URINE APTIMA COMBO 2 TESTING}

Melanie Bissessor*. Melbourne Sexual Health Centre, Sexual Health, Carlton, Australia

\subsection{6/sextrans-2019-sti.692}

Background In a previous study of men attending Melbourne Sexual Health Centre who had N. gonorrhoeae detected by urine Aptima Combo 2 (AC2) testing, 11\% were asymptomatic, reporting no urethral symptoms. This study aimed to determine if $N$. gonorrhoeae can be cultured from asymptomatic men screening positive for $N$. gonorrhoeae by nucleic acid amplification testing (NAAT) of urine.

Methods Between 1 July 2017 and 30 September 2018, men reporting sex with men attending Melbourne Sexual Health Centre who did not report urethral symptoms were screened for N. gonorrhoeae by AC2 testing of urine. NAAT positive men were recalled and a urethral swab performed for gonococcal culture using modified Thayer Martin media with determination of minimum inhibitory concentrations (MICs) for penicillin, azithromycin, ceftriaxone and ciprofloxacin by agar dilution.

Results There were 612 cases (538 individuals) positive for $N$. gonorrhoeae by urine AC2: 548 (90\%) reported urethral symptoms; 64 (10\%) did not report symptoms. Thirteen asymptomatic cases were excluded because of antibiotic use at or following screening. Of the remaining 51 asymptomatic men, 25 (49\%) had a urethral swab performed a median of 4 days after screening. Thirteen men had urethral discharge at the return visit, 7 of whom reported the discharge at the return visit. Of the 25 men who were swabbed, 18 (72\%) were culture positive for $N$. gonorrhoeae. Among the 12 men who remained asymptomatic with no discharge at the return 